\title{
Una clase de modelos bioeconómicos continuos*
}

\author{
Eduardo González-Olivares \\ Instituto de Matemáticas, Universidad Católica de Valparaíso \\ Casilla 4059, Valparaíso, Chile \\ E-mail: ejgonzal@ucv.cl
}

\begin{abstract}
RESUMEN. En este trabajo se desarrolla el análisis cualitativo de la estabilidad de una clase de modelos bioeconómicos de tiempo continuo, que representan la explotación de una pesquería de acceso abierto, modificando la versión dinámica del modelo de Schaefer, propuesta por Smith (1969). Se introduce una función de captura que es más realista que la usada en la hipótesis de captura por unidad de esfuerzo (Schaefer, 1954), puesto que toma en cuenta la saturación de la captura cuando la biomasa crece. Se prueba la existencia de equilibrios bioeconómicos, el autocontrol de la pesquería de acuerdo a los cambios en los parámetros, se discute la validez de la aplicabilidad de los modelos y se presentan algunas simulaciones realizadas con el programa PHASER.
\end{abstract}

Palabras claves: Capturabilidad, función de producción, esfuerzo de pesca, pesquerías de acceso abierto, equilibrio bionómico, estado estable.

\section{A class of continuos bioeconomic models}

\begin{abstract}
In this work a qualitative stability analysis of a continuous time bioeconomic model is developed representing the exploitation of an open acces fisheries, by modifying the dynamic version proposed by Smith (1969) to the Schaefer model. It introduces a catch-rate function that is more realistic than the used in catch per unit effort hypothesis (Schaefer, 1954), because harvesting saturation is taken into account when biomass increases. The existence of bioeconomic equilibriums and self-control of the fishery are proved according to changes in parameters, the validity of models applicability is discussed and simulations with PHASER package are shown.
\end{abstract}

Key words: Catchability, production function, fishing effort, open-access fishery, bionomic equilibrium, steady state.

\section{INTRODUCCION}

El problema de las pesquerías de acceso abierto ha sido bastante estudiado por muchos autores a partir de la teoría económica desarrollada por Gordon (1954) y se han formulado diferentes modelos matemáticos. En este trabajo se estudia una clase de modelos microeconómicos deterministas, considerando la versión dinámica propuesta por Smith (1969), al modelo de Schaefer (1954), también conocido como modelo Gordon-Schaefer (Clark, 1990) y su orientación es principalmente teórica.

\footnotetext{
* Financiado por Proyecto D.G.I.P. UCV 124 753-97.
}

En este modelo se describe la explotación de una pesquería de acceso abierta compuesta por una sola especie en la cual, tanto el tamaño de la población como el esfuerzo de pesca varían continuamente con el tiempo; se demuestran algunos aspectos del estado estable y se da una interpretación bioeconómica de los resultados.

Para esta clase de modelos se considera una función de captura diferente a la usada en la conocida hipótesis de «captura por unidad de esfuerzo» o Hipótesis de Schaefer. La dinámica de la población de peces, es representada por la ecuación de crecimiento logístico. 
En la mayoría de los modelos aplicados en el manejo de recursos renovables se considera el esfuerzo de pesca como un parámetro más. Al considerar el esfuerzo de pesca como una variable que cambia continuamente en el tiempo, se obtiene un sistema de ecuaciones diferenciales ordinarias no lineales, que se analiza principalmente en el primer cuadrante donde tiene sentido biológico. El sistema obtenido coincide con modelos de depredación, y se puede considerar que la especie explotada es la presa y la depredación es la acción del esfuerzo de pesca realizado por el hombre.

Aunque la aplicabilidad de estos modelos (modelos estratégicos) al manejo de recursos pesqueros puede ser cuestionada por no considerar la incerteza de los datos, ellos entregan un marco teórico para la discusión de estos problemas bioeconómicos y además pueden aplicarse a una clase más amplia de fenomenos biológicos, como es el caso de las interacciones entre poblaciones (May, 1974; Yodzis, 1989). La principal diferencia con los modelos tácticos o de simulación es que éstos requieren una gran y detallada cantidad de información de la especie involucrada (Hilborn y Walters, 1992). Esta controversia usual entre los modeladores, nos parece inadecuada, ya que ambos modos de asumir la realidad tienen ventajas y desventajas, especialmente en lo que se refiere al manejo de recursos renovables.

\section{PROPOSICION DEL PROBLEMA}

Tratando de encontrar una medida para la actividad pesquera fue desarrollado por los biólogos pesqueros el concepto de esfuerzo de pesca (Hannensson, 1993). Suponiendo que esa medida puede ser determinada, la hipótesis de Schaefer establece que la captura por unidad de esfuerzo (C.P.U.E.) es un índice directamente proporcional a la abundancia del stock (Clark, 1985) .

Se obtiene así, la conocida relación, $\mathrm{H}(\mathrm{x}, \mathrm{E})=\mathrm{q}$ $\mathrm{x} \mathrm{E}$, donde la función $\mathrm{H}(\mathrm{x}, \mathrm{E})$ es la tasa de cosecha o captura del recurso o función de producción (Clark, 1979, 1990), q > 0, es el coeficiente de capturabili-dad, $\mathrm{x}=\mathrm{x}(\mathrm{t})$ denota el tamaño del stock de la población o la fracción explotada de la biomasa de peces en el tiempo $\mathrm{t}$, mientras que $\mathrm{E}=\mathrm{E}(\mathrm{t})$ indica el esfuerzo nominal de pesca, una medida de la actividad pesquera, que podría estar representado por el número de embarcaciones activas en la pesquería en el tiempo $t$ (Clark, 1979; Smith, 1969), o en otros casos con información más detallada sobre el tamaño de redes, líneas o trampas tiradas.

Si bien se puede hacer una diferencia entre los conceptos de mortalidad por pesca, esfuerzo nominal de pesca y esfuerzo de pesca (Clark, 1985), para el estudio que aquí se realiza no es necesario hacer esta distinción. En resumen, el esfuerzo de pesca transforma las variables biológicas de crecimiento, mortalidad y reclutamiento en captura y representa la variable controlable que puede ser manipulada para optimizar el rendimiento (Rothschild, 1977).

Como el tamaño del stock $\mathrm{x}$, usualmente no es observable y los datos de captura y esfuerzo pueden ser obtenidos para cada pesquería, la relación anterior entrega una evaluación para el stock. Sin embargo, la hipótesis de Schaefer tiene al menos dos limitaciones (Clark, 1985):

a) el valor del coeficiente de capturabilidad q, no puede ser estimado a menos que se tenga una evaluación independiente del stock, y

b) la ecuación $\mathrm{H}(\mathrm{x}, \mathrm{E})$ = q x E es sólo una hipótesis y como tal puede resultar un estimador sesgado.

De hecho esta hipótesis es engañosa, especialmente para las pesquerías de especies pelágicas y por ello en algunas referencias (Clark, 1990; Hannensson, 1993; Walters, 1986) se propone que para tener una relación «más realista», es conveniente asumir que la función de cosecha o captura sea del tipo

$$
H(x, E)=q x^{a} E^{b}
$$

con $0<\mathrm{a}, \mathrm{b} £ 1$, conocidas en Economía como funciones de producción tipo Cobb-Douglas o relaciones log-lineal.

Usando este tipo de funciones de producción se han desarrollado modelos que proporcionan una aplicación empírica, basados en el análisis de algunas pesquerías, con especial preocupación del problema de la extinción del stock en un sistema de acceso abierto (Amudsen et al., 1995; Bjørndal y Conrad, 1987; Opsomer y Conrad, 1994), pero su dinámica ha sido sólo estudiada parcialmente (Cheng et al., 1981; González-Olivares y OsorioSantibáñez, 1997). Sin embargo, desde nuestra persectiva no existen modelos más o menos realistas, sino que existen modelos más o menos adecuados a un determinado fenómeno biológico o bioeconómico, porque en ellos no existen leyes empíricas que lo rijan.

Clark (1990) considera $b=1$ e indica que: $«$ la 
teoría no se complica si $\mathrm{x}^{\mathrm{a}}$ es reemplazada por una función $\mathrm{G}(\mathrm{x})$ no decreciente arbitraria, la cual puede muchas veces ser más realista. La suposición que el coeficiente $G(x)$ sea no decreciente es claramente deseable, pues implica que la tasa de captura correspondiente a un esfuerzo dado $E$ no puede decrecer si el nivel de población se incrementa». Una tasa de captura o función de producción que también satisface este propósito está dada por:

$$
H(x, E)=\frac{q x E}{a+l x}
$$

Las constantes a y $l$ son ambas positivas y tienen unidades de medida adecuadas que permiten la suma de las variables involucradas y $l$ puede asumirse igual a 1 sin perder generalidad. Aquí el cuociente $\mathrm{q} / l$ representa la tasa máxima de captura por unidad de esfuerzo, es decir, es la máxima biomasa que puede ser capturada por cada unidad de esfuerzo en cada unidad de tiempo y el cuociente a/l indica la cantidad de biomasa necesaria para alcanzar la mitad del valor de la tasa máxima. La función $\mathrm{H}$ propuesta para la tasa de captura, es lineal respecto a $\mathrm{E}$, permitiendo una manipulación más fácil para la optimización del esfuerzo; además, admite dos interpretaciones diferentes:

a) Si se considera la tasa de captura como $\mathrm{H}(\mathrm{x}, \mathrm{E})=$ q $\mathrm{G}(\mathrm{x}) \mathrm{E}$, donde

$$
G(x)=\frac{x}{a+l x} \text { y cuya derivada, } G^{\prime}(x)=\frac{a}{(a+l x)^{2}}
$$

es positiva, se concluye que no existe una concentración constante de la biomasa. Esta es una suposición razonable porque indica que existe una cota superior para la tasa de depredación (captura de la biomasa), cuando se completa la capacidad de la industria pesquera o se satisface la demanda de los industriales pesqueros.

b) Si la tasa de captura se escribe como $\mathrm{H}(\mathrm{x}, \mathrm{E})=$ $\mathrm{Q}(\mathrm{x}) \mathrm{x}$ E, donde la función:

$$
Q(x)=\frac{q}{a+l x}
$$

expresa una modificación al coeficiente de capturabilidad q, considerado constante en la hipótesis de Schaefer. Claramente, $Q(x)$ varía inversamente proporcional a la abundancia del recurso, esto es, decrece cuando la biomasa crece y recíprocamente. Se debe hacer notar también que esta función de producción $\mathrm{H}(\mathrm{x}, \mathrm{E})=\mathrm{Q}(\mathrm{x}) \mathrm{x} \mathrm{E}$, toma en cuenta la idea de saturación cuando la biomasa crece, esto es

$$
\text { Si } x \circledast ¥ \text { entonces } \mathrm{H}(x, \mathrm{E}) \circledast \frac{\mathrm{qE}}{l}
$$

$\mathrm{Al}$ asumir que el cambio en el nivel del esfuerzo de pesca $\mathrm{E}$ varía proporcionalmente con el beneficio neto percibido por la industria pesquera en cualquier instante $\mathrm{t}$ (Smith, 1969), se tiene que,

$$
E^{\prime}=b(p H(x, E)-c E)
$$

donde $\mathrm{p}, \mathrm{q}$ y $\mathrm{b}$ son constantes positivas, en que $\mathrm{p}$ representa el precio de la biomasa de peces desembarcada, c es el costo del esfuerzo de pesca y b, llamado parámetro de rigidez o de ajuste (Amudsen et al., 1995; Bjørndal y Conrad, 1987), y representa la capacidad de reacción de la industria pesquera (Smith, 1969).

Si $F(x)$ expresa la función de crecimiento poblacional de la especie, se obtiene para la pesquería de acceso abierto o de propiedad común, un modelo descrito por un sistema dinámico constituido por dos ecuaciones diferenciales no-lineales:

$$
\begin{aligned}
x^{\prime} & =F(x)-\frac{q x E}{a+l x} \\
E^{\prime} & =b\left[\frac{p q x}{a+l x}-c\right] E
\end{aligned}
$$

Este sistema corresponde a un modelo de depredación, en que la función de captura corresponde a una respuesta funcional del depredador Holling tipo 2 (Murray 1989; May, 1974; Sánchez-Garduño, 1990; Yodzis 1989), y que han sido propuestos para evitar la principal deficiencia del modelo LotkaVolterra, ya que este es estructuralmente inestable, pues tiene un punto de equilibrio no trivial, alrededor del cual hay sólo oscilaciones que dependen de las condiciones iniciales (May, 1974; Murray, 1989).

En este trabajo se analiza principalmente en el primer cuadrante $\mathrm{G}=\left(\mathrm{R}^{+}{ }_{0}\right)^{2}$, donde tiene un claro sentido biológico. Goh (1980) usando una función de Liapunov adecuada, demuestra que el sistema (3) es globalmente estable si tiene un único equilibrio positivo en el primer cuadrante y verifica una determinada desigualdad. Sin embargo, no da condiciones para la existencia de oscilaciones que son comunes en las interacciones de depredación como es la situación que aquí se analiza.

Una clase particular de modelos se tiene cuando $\mathrm{F}(\mathrm{x})$ es la ley logística de crecimiento (o de Verlhust), obteniéndose el sistema:

$$
x^{\prime}=\left[r\left(1-\frac{x}{K}\right)-\frac{q E}{a+l x}\right] x
$$




$$
E^{\prime}=b\left[\frac{p q x}{a+l x}-c\right] E
$$

donde $\mathrm{K}$ representa la capacidad de soporte del medio ambiente y $\mathrm{r}$ es la tasa intrínseca de crecimiento de la población. La función logística ha sido también fuertemente criticada por cuanto la curva de producción del stock (curva de rendimiento en el equilibrio) alcanza su máximo exactamente en la mitad del tamaño máximo teórico del stock o biomasa (Pella y Tomlinson, 1969) y por eso se propone que la curva sea asimétrica y en algunas de ellas el máximo se alcanza en un punto en que el tamaño del stock está por debajo de la mitad de su valor máximo (capacidad de soporte). El sistema (4) tiene como puntos de equilibrio o singularidades a $\mathrm{P}_{0}=(0,0), \mathrm{P}_{1}=(\mathrm{K}, 0)$ y el punto no trivial $\mathrm{P}=$ $\left(\mathrm{x}_{\mathrm{e}}, \mathrm{E}_{\mathrm{e}}\right)$, es el punto de intersección de (las isoclinas)

la recta $x=\frac{a c}{p q-c l}$ y la parábola $E=\frac{r}{q}\left(1-\frac{x}{K}\right)(a+l x)$

Agrupando los ocho parámetros y redefiniendo las variables, se puede simplificar el sistema (4) a un sistema polinomial «adimensional» que tiene sólo tres parámetros, y el cual es cualitativamente equivalente (Andronov, 1973; Sotomayor, 1979); este es descrito por las ecuaciones:

$$
\begin{aligned}
& x^{\prime}=[(1-x)(A+x)-E] x \\
& E^{\prime}=B[x-(A+x) C] E \\
& \operatorname{con} A=\frac{a}{l k}, B=\frac{b p q}{l r}, C=\frac{c l}{p q}
\end{aligned}
$$

Reescalando el tiempo, la relación entre las antiguas y nuevas variables, está dado por:

$$
t=\frac{r}{A+x} t, x_{(a)}=K x_{(n)} y E_{(a)}=\frac{K l r}{q} E_{(n)}
$$

Los subíndices $\mathrm{n}$ y a indican a las nuevas y antiguas variables.

Claramente, las tres constantes A, B y C resultan también positivos y para un estudio cualitativo no es necesario conocer cada uno de los ocho parámetros originales, sino que es suficiente establecer o conocer las relaciones existentes entre ellos.
Nótese que la nueva variable $\mathrm{x}$ indica la fracción de la biomasa y tiene su dominio entre 0 y 1 .

\section{PRINCIPALES RESULTADOS}

El sistema (5) tiene como puntos fijos a $\mathrm{P}_{0}=(0,0)$, $\mathrm{P}_{1}=(1,0)$ y el equilibrio no trivial $\mathrm{P}_{\mathrm{e}}=\left(\mathrm{x}_{\mathrm{e}}, \mathrm{E}_{\mathrm{e}}\right)$ está dado por:

$$
x_{e}=\frac{A C}{1-C} y E_{e}=\frac{(1-C-A C) A}{(1-C)^{2}}
$$

mientras que la traza de la matriz jacobiana $\mathrm{M}_{\mathrm{e}}$ es

$$
\operatorname{Tr}_{e}=\frac{(1-C-A-A C) A C}{(1-C)^{2}}
$$

En términos de los parámetros originales tenemos los siguientes resultados:

\section{Teorema 1}

Si a c > K (p q - c 1), el sistema (4) tiende al punto $(\mathrm{K}, 0)$, esto es, la biomasa alcanza siempre su máximo valor $\mathrm{K}$, independiente del esfuerzo inicial $\mathrm{E}$ (Fig. 1).

Esto significa que la pesquería de la especie considerada no es rentable y eso provoca el éxodo de los barcos de la industria, los que podrían destinarse a otra actividad más rentable. Matemáticamente, supuesto que a c > K (p q - c 1) y si p q < c 1, el punto fijo no trivial $\mathrm{P}_{\mathrm{e}}$ está en el tercer cuadrante, mientras que está en el cuarto cuadrante si p q >c 1 .

Cuando el punto de equilibrio no trivial $\mathrm{P}_{\mathrm{e}}$ está en el primer cuadrante, coexiste con los puntos $(0,0)$ $\mathrm{y}(\mathrm{K}, 0)$; se cumple simultáneamente que $\mathrm{p} \mathrm{q}>\mathrm{c} 1 \mathrm{y}$ a c $<\mathrm{K}$ (p q - c 1). El signo de la traza de la matriz jacobiana en $\mathrm{P}_{\mathrm{e}}$ depende del factor $\mathrm{T}=1$ - $\mathrm{C}-\mathrm{A}$ $\mathrm{AC}$ y entonces tenemos las siguientes afirmaciones:

\section{Teorema 2}

Si $\operatorname{TrM}_{\mathrm{e}}=0$, se cumple que c $1(1 \mathrm{~K}+\mathrm{a})=\mathrm{p} \mathrm{q}(1 \mathrm{~K}$ - a) que en el sistema (4) el punto $P_{e}$ es un foco débil.

Esto significa que la biomasa y el esfuerzo oscilan alrededor de $\left(\mathrm{x}_{\mathrm{e}}, \mathrm{E}_{\mathrm{e}}\right)$, pero lentamente tiende a estabilizarse en ese punto (Fig. 2). 


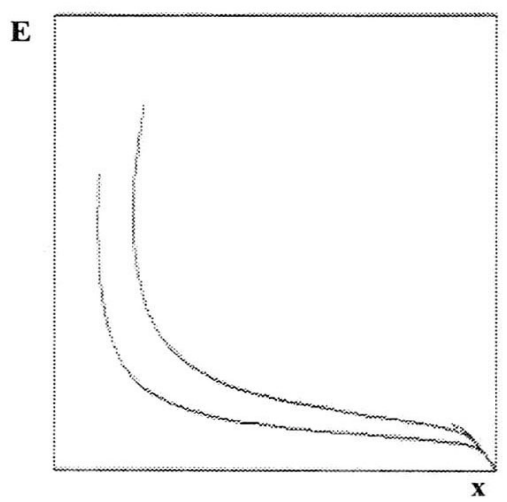

Figura 1. Para $A=0.5, B=1$ y $C=0.75$, el punto $P_{1}=$ $(1,0)$ del sistema (5) es atractor. En la región $K=$ $\left\{(\mathbf{x}, E) \in\left(R_{0}^{+}\right)^{2} / x £ 1\right\}$ del plano de fase, se muestran tres órbitas con condiciones iniciales en los puntos $(0.9$, $0.1) ;(0.1,0.65)$ y $(0.2,0.8)$. La relación costo precio es alta, es decir, la especie considerada no es rentable y el esfuerzo tiende a cero.

Figure 1. For $A=0.5, B=1$ and $C=0.75$, the point $P_{1}=$ $(1,0)$ of system $(5)$ is an attractor. On region $K=\{(x, E)$ $\left.\in\left(\mathbf{R}_{0}^{+}\right)^{2} / \mathbf{x} £ 1\right\}$ of phase plane, three orbits with initial conditions at $(0.9,0.1) ;(0.1,0.65)$ and $(0.2,0.8)$ are showed. The cost-price relation is high, i.e, the specie considered is not profitable and fishing effort tends to zero.

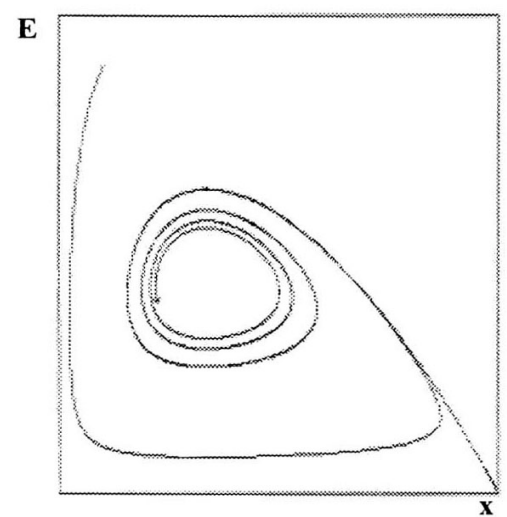

Figura 2. Para $A=0.333333, B=1$ y $C=0.5$, el punto $P_{e}=(0.333333,0.444444)$ del sistema (5) es atractor débil. En la región $K=\left\{(x, E) \in=\left(R_{0}^{+}\right)^{2} / x £ 1\right\}$ del plano de fase, se muestran las órbitas con condiciones iniciales en $(0.995,0.005)$; $(0.1,0.9)$ y $(0.333333$, 0.444444). La biomasa y el esfuerzo oscilan alrededor de $P_{e}$, $y$ lentamente tienden a ese punto.

Figure 2. For $\mathrm{A}=0.333333, \mathrm{~B}=1$ and $\mathrm{C}=0.5$, the point $P_{e}=(0.333333,0.444444)$ of system (5) is a weak attractor. Orbits with initial conditions at $(0.995$, $0.005) ;(0.1,0.9)$ and $(0.333333,0.444444)$ are showed. The biomass and fishing effort oscillate surround $P_{e}$, and slowly tend to this equilibrium point.

\section{Teorema 3}

Si $\operatorname{TrM}_{e}>0$ se tiene que c $1(1 \mathrm{~K}+\mathrm{a})>\mathrm{pq}(1 \mathrm{~K}-\mathrm{a})$, el sistema (4) tiene un ciclo límite y el punto $\mathrm{P}_{\mathrm{e}}$ es inestable (Fig. 3).

Esto implica que no existe un equilibrio bionómico (Clark, 1990) y la pesquería puede autorregularse, es decir, el esfuerzo de pesca es regulado por la disminución y aumento de la biomasa, oscilando esta alrededor del valor $\mathrm{x}_{\mathrm{e}} \mathrm{y}$ el esfuerzo alrededor de $\mathrm{E}_{\mathrm{e}}$ dadas en la relación (7).

\section{Teorema 4}

Si $\operatorname{TrM}_{\mathrm{e}}<0$ entonces, c $1(1 \mathrm{~K}+\mathrm{a})<\mathrm{p} \mathrm{q}(1 \mathrm{~K}-\mathrm{a})$ $y$ el punto $\mathrm{P}_{\mathrm{e}}$ es estable (Fig. 4).

La pesquería tiende en el largo plazo a estabilizarse al punto de equilibrio no trivial $\left(\mathrm{x}_{\mathrm{e}}, \mathrm{E}_{\mathrm{e}}\right)$.

\section{CONCLUSIONES}

El modelo de Smith tiempo continuo basado en la hipótesis de Schaefer, es la primera versión dinámica que modela una pesquería de acceso abierto, considerando que el esfuerzo nominal de pesca, representado por el número de barcos en la pesquería, varía en forma continua y usa una simple ecuación para modelar la dinámica de la flota, $E^{\prime}=b \mathrm{p}$, donde $\mathrm{p}$ representa la ganancia de la industria pesquera en el tiempo t.

Se sabe que el sistema dinámico así formado, cuando tiene un punto de equilibrio (equilibrio bionómico) no trivial en el primer cuadrante, es (un nodo) siempre globalmente estable (Goh, 1980; Clark, 1990; González-Olivares, 1991), lo cual implica que en el largo tiempo la pesquería se mantendría en un equilibrio fijo, lo que a juicio de algunos modeladores sería irrealista, ya que son conocidas las fluctuaciones que han tenido diversas pesquerías.

Desde el punto de vista bioeconómico, la clase de modelos analizados que modifican la tasa de captura propuesta por Schaefer, pero siguen el razonamiento de Smith respecto a la dinámica del esfuerzo, recogen algunas de las observaciones formuladas a este modelo, y muestran que la estabilidad de la pesquería de libre acceso, depende fundamentalmente del valor de la traza de la matriz jacobiana, la que a su vez depende principalmente de los valores A y C, siendo más importante para predecir el comportamiento futuro del sistema las relaciones entre los parámetros que los valores particulares de cada uno. 


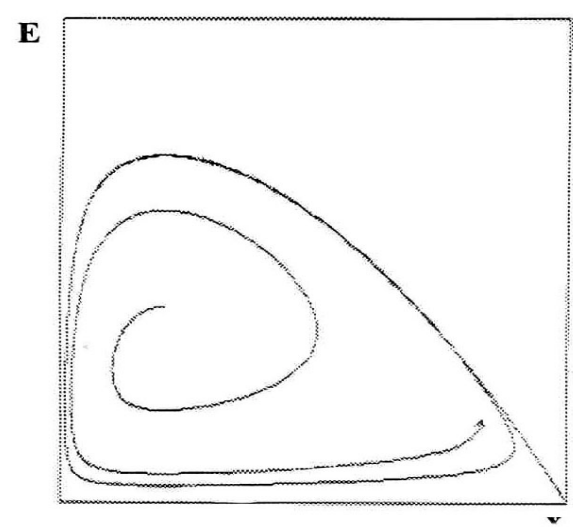

Figura 3. Para $A=0.2, B=1$ y $C=0.5$, el punto $P_{e}=$ $(0.2,0.32)$ del sistema (5) es repulsor, asegurándose la existencia de un ciclo límite. En la región $K=\{(x, E) \in$ $\left.\left(\mathbf{R}_{0}^{+}\right)^{2} / \mathbf{x} £ 1\right\}$ del plano de fase, se muestran las órbitas con condiciones iniciales en $(0.995,0.005) ;(0.2$, 0.4) y $(0.2,0.32)$. La pesquería puede autorregularse, o sea, el esfuerzo de pesca y la biomasa oscilan alrededor del punto $P_{\mathrm{e}}$.

Figure 3. For $\mathrm{A}=0.2, \mathrm{~B}=1$ and $\mathrm{C}=0.5$, the point $\mathrm{P}_{\mathrm{e}}=$ $(0.2,0.32)$ of system (5) is a repellor and a limit cycle exist. Orbits with initial conditions at $(0.995,0.005)$; $(0.2,0.4)$ and $(0.2,0.32)$ are showed. The fishery can be autoregulate, i.e., fishing effort and biomass oscillate surround $P$.

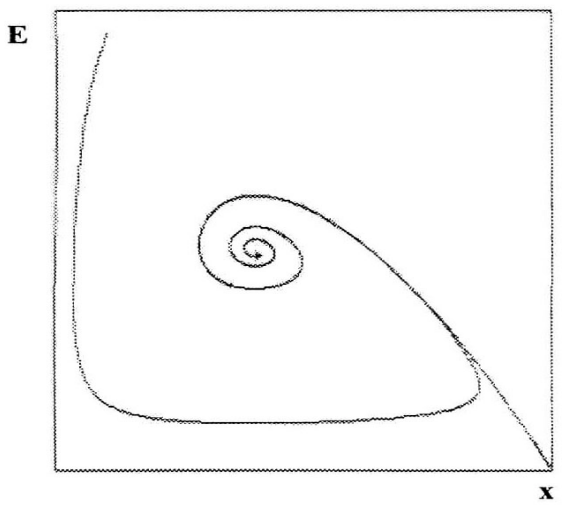

Figura 4. Para $A=0.4, B=1$ y $C=0.5$, el punto $P_{e}=$ $(0.4,0.48)$ del sistema (5) es atractor. En la región $K=$ $\left\{(\mathbf{x}, \mathbf{E}) \in\left(\mathbf{R}_{0}^{+}\right)^{2} \mathbf{x} £ 1\right\}$ del plano de fase, se muestran las órbitas con condiciones iniciales en $(0.995,0.005)$; $(0.1,0.95)$ y $(0.4,0.48)$. La pesquería tiende en el largo plazo a estabilizarse al punto de equilibrio no trivial $\mathbf{P}_{\mathrm{e}}$.

Figure 4. For $\mathrm{A}=0.4, \mathrm{~B}=1$ and $\mathrm{C}=0.5$, the point $\mathrm{P}_{\mathrm{e}}=$ $(0.4,0.48)$ of system $(5)$ is an attractor. Orbits with initial conditions at $(0.995,0.005) ;(0.1,0.95)$ and $(0.4$, 0.48) are showed. The fishery tends to non-trivial equilibrium point $\mathbf{P}_{\mathrm{e}}$.
El sistema (4) representaría adecuadamente lo que acontece en algunas pesquerías de una sola especie y de acceso abierto, pues predice diversas situaciones para diferentes valores de los parámetros, como es el caso de las pesquerías artesanales, donde la pesca se realiza intensamente en los períodos de mayor abundancia y disminuye en aquellas etapas en que el recurso está en sus más bajos niveles.

Si la relación costo-precio es alta, el sistema tiende a la singularidad a $\mathrm{P}_{1}=(\mathrm{K}, 0)$, es decir, los industriales estarían retirándose de la pesquería porque el negocio no es conveniente y la especie tiende a preservarse en el valor máximo de la biomasa (la capacidad de soporte); esta situación se da matemáticamente cuando no existe un equilibrio en el primer cuadrante.

Si la traza es cero, el sistema tiene un foco débil atractor. Esta situación implica que a largo plazo la pesquería tiende al punto de equilibrio no trivial $\mathrm{P}_{\mathrm{e}}$. Además, desde un punto de vista práctico es difícil la manipulación de los parámetros para preservar esta igualdad.

Si la traza es positiva se tiene la existencia de un ciclo límite, lo que indica que el esfuerzo de los pescadores se autorregula por la disponibilidad de la biomasa (no existe equilibrio bionómico), habiendo períodos de auge y otros de depresión en la pesquería. Aquí puede suceder que el punto de equilibrio no trivial tenga un valor pequeño respecto a $\mathrm{x}$, y la amplitud del ciclo sea grande, por lo cual se corre el riesgo de agotar el stock, y por consecuencia, el término de la pesquería.

El sistema tiende al punto de equilibrio, $\mathrm{P}_{\mathrm{e}}=\left(\mathrm{x}_{\mathrm{e}}\right.$, $\mathrm{E}_{\mathrm{e}}$ ), cuando el costo por unidad de esfuerzo es mucho menor que el precio de la unidad de biomasa desembarcada. Ello implica que el negocio es atractivo para los inversionistas y entrarían nuevos barcos a la pesquería, pero a medida que transcurre el tiempo la pesquería se mantendría en equilibrio, tal como lo predice el modelo de Smith.

La clase de modelos presentados no toman en cuenta los cambios en el tamaño de stock a causa de fenómenos estacionales (Ardito et al., 1993) o de la acción del hombre como depredador, el cual mejorando la tecnología para realizar la pesca, aumenta la cantidad de peces capturados. Esta situación, que ha provocado gran daño en ciertas poblaciones, dado que la tasa reproductiva de la especie es más lenta que los cambios que introduce la industria pesquera para ser más eficiente, no es prevista por esta clase de modelos. 
Algunas modificaciones que se pueden introducir a estos modelos para hacerlos más adecuados a cierto tipo de pesquerías, es asumir que el precio $\mathrm{p}$ y el costo c sufren fluctuaciones estacionales, esto es, son funciones conocidas del tiempo (Ardito et al., 1993) u otras como serían considerar que el precio es dependiente del stock, o bien, adicionando el efecto de la depredación por parte de otra especie (Spencer, 1997), pero en algunos casos se daría origen a modelos que podrían resultar bastante más complicados matemáticamente.

Aunque se sostenga que los modelos deterministas no son realistas (Clark, 1985; Hilborn y Walters, 1992), debido principalmente a que el manejo de los recursos pesqueros opera con un alto grado de incertidumbre, se estima que la clase de modelos teóricos como el que aquí se presenta, muestra los aspectos esenciales de los problemas que afectan este manejo. Puesto que los stock de peces de las principales pesquerías no pueden ser observados directamente, las estimaciones del pasado y presente de los niveles de abundancia del stock son a menudo incompletas y no confiables y muchas veces, en base de esos datos imprecisos se estiman parámetros, se toman medidas de manejo, se determinan los niveles de captura y se desechan modelos sin tomar debida cuenta si son razonables sus predicciones.

\section{APÉNDICE}

Se realiza el análisis local de los puntos de equilibrio del sistema (5), $\mathrm{P}_{\mathrm{o}}=(0,0), \mathrm{P}_{1}=(1,0)$ y $\mathrm{P}_{\mathrm{e}}=\left(\mathrm{x}_{\mathrm{e}}\right.$, $\mathrm{E}_{\mathrm{e}}$ ), el punto de equilibrio no trivial dado por la relación (7), y que es el punto de intersección entre

la recta $x=\frac{A C}{1-C}$ y la parábola $E=(1-x)(A+x)$

considerando principalmente el comportamiento en el primer cuadrante $\mathrm{G}=\left(\mathrm{R}_{\mathrm{o}}^{+}\right)^{2}$, (porque ahí tiene un claro sentido biológico), de acuerdo a los signos de la parte real de los valores propios de la matriz jacobiana (o matriz de la comunidad) del sistema. Para que $\mathrm{P}_{\mathrm{e}}$ esté en $\mathrm{G}$, debe cumplirse simultáneamente que $1-\mathrm{C}-\mathrm{A} \mathrm{C}>0$ y $1-\mathrm{C}>0$. Se define los siguientes subconjuntos del espacio de parámetros $\left(\mathrm{R}^{+}\right)^{3}$.

$$
\mathrm{Q}_{\mathrm{e}}=\left\{(\mathrm{A}, \mathrm{B}, \mathrm{C}) \in\left(\mathrm{R}^{+}\right)^{3} / \mathrm{C}<1^{\wedge} \mathrm{A} \mathrm{C}<1-\mathrm{C}\right\}
$$

$$
\begin{aligned}
& \mathrm{Q}_{3}=\left\{(\mathrm{A}, \mathrm{B}, \mathrm{C}) \in\left(\mathrm{R}^{+}\right)^{3} / \mathrm{C}>1^{\wedge} \mathrm{A} \mathrm{C}>1-\mathrm{C}\right\} \\
& \mathrm{Q}_{4}=\left\{(\mathrm{A}, \mathrm{B}, \mathrm{C}) \in\left(\mathrm{R}^{+}\right)^{3} / \mathrm{C}<1^{\wedge} \mathrm{A} \mathrm{C}>1-\mathrm{C}\right\}
\end{aligned}
$$

Es claro que, dependiendo en cual de los subconjuntos estén los parámetros, ocurren diferentes comportamientos del sistema. En $\mathrm{Q}_{\mathrm{e}}$, tres singularidades coexisten en $\mathrm{G}$, pero en $\mathrm{Q}_{3}$ y $\mathrm{Q}_{4}$, sólo hay dos singularidades en el primer cuadrante $\left(\mathrm{P}_{1}\right.$ y $\left.\mathrm{P}_{0}\right)$. La matriz jacobiana del campo (5) es

$$
M=\left[\begin{array}{lr}
A+2(1-A) x-3 x^{2}-E-x \\
B(1-C) E & B(1-C) x-A C
\end{array}\right]
$$

\section{CASO $\mathrm{Q}_{3}$}

En este caso, el punto de equilibrio no trivial $\mathrm{P}_{\mathrm{e}}$ está en el tercer cuadrante. Es claro que si los parámetros están en $\mathrm{Q}_{3}$, la región $\mathrm{K}=\left\{(\mathrm{x}, \mathrm{E}) \in\left(\mathrm{R}_{0}^{+}\right)^{2} / \mathrm{x} £ 1\right\}$ del espacio de fase, es positivamente invariante para cualquier $\mathrm{E}_{0}$, puesto que:

1) En la ecuación $5 b$, el término $(1-C) x-A C$ es negativo,

2) los ejes $\mathrm{x}=0 \mathrm{y} \mathrm{E}=0$ son variedades invariantes del sistema (5)

3) en general (independiente de los parámetros), cualquier trayectoria cruza la línea $\mathrm{x}=1$, hacia el interior de K.

En este caso, el eje $\mathrm{x}$ es una variedad repulsora para el origen $(0,0)$. Sigue por el Teorema de Poincaré-Bendixon (Arrowsmith y Place, 1992) que $\mathrm{P}_{1}=(1,0)$ es un punto de equilibrio global asintóticamente estable de la región $\mathrm{K}$, para cualquier combinación de los parámetros.

\section{CASO $Q_{4}$}

El equilibrio no trivial $\mathrm{P}_{\mathrm{e}}=\left(\mathrm{x}_{\mathrm{e}}, \mathrm{E}_{\mathrm{e}}\right)$ está en el cuarto cuadrante $\mathrm{y}_{\mathrm{e}}>1$, es decir, habría más población en el equilibrio que la capacidad de soporte; la región $\mathrm{K}=\left\{(\mathrm{x}, \mathrm{E}) \in\left(\mathrm{R}_{0}^{+}\right)^{2} / \mathrm{x} £ 1\right\}$, es invariante en el espacio de fase; las órbitas cruzan hacia el interior de $\mathrm{K}$ a través de la recta $\mathrm{x}=1$ y la expresión $(1-\mathrm{C})$ $\mathrm{x}$ - A C es negativa, pues $(1-\mathrm{C}) \mathrm{x}<1-\mathrm{C}<\mathrm{A} \mathrm{C}$. Al considerar los valores propios de la matriz jacobiana, resulta que $(0,0)$ es punto silla, mientras que $(1,0)$ es atractor global. Esto nos indica que especie explotada alcanza su máximo nivel y la pesquería no es rentable. 


\section{CASO $Q_{\mathrm{e}}$}

$\mathrm{Al}$ tomar valores de los parámetros en $\mathrm{Q}_{\mathrm{e}}$ se deduce también que la región $\mathrm{K}=\left\{(\mathrm{x}, \mathrm{E}) \in\left(\mathrm{R}_{0}^{+}\right)^{2} / \mathrm{x} £ 1\right\}$, es positivamente invariante en el espacio de fase, $i$. e., toda trayectoria del sistema que comienza en $\mathrm{K}$, permanece allí.

1) $\mathrm{Si} \operatorname{TrM}_{e}<0$, los puntos $\mathrm{P}_{0}=(0,0)$ y $\mathrm{P}_{1}=(1,0)$ son puntos sillas y $\mathrm{P}_{\mathrm{e}}=\left(\mathrm{x}_{\mathrm{e}}, \mathrm{E}_{\mathrm{e}}\right)$ es global asintóticamente estable en la región $\mathrm{K}$, porque la posibilidad de ciclos límites es eliminada aplicando el test de Dulac (Arrowsmith y Place, 1992; Clark, 1990) con la función $\mathrm{G}(\mathrm{x}, \mathrm{E})=\mathrm{x}^{\mathrm{m}} \mathrm{E}^{\mathrm{n}}$, siendo

$$
m=\frac{1+C-2 A C}{A C-1} \quad y \quad n=\frac{1+A-B-A B C}{A C-1}
$$

2) $\mathrm{Si} \operatorname{TrM}_{e}>0$, el punto $P_{e}$ es repulsor, mientras que $\mathrm{P}_{1}=(1,0)$ y $\mathrm{P}_{0}=(0,0)$ son ambos puntos sillas; por teorema de Poincaré-Bendixon existe al menos una órbita periódica alrededor de $\mathrm{P}_{\mathrm{e}}$.

3) $\mathrm{Si} \operatorname{Tr} \mathrm{M}_{\mathrm{e}}=0$, se demuestra la existencia de órbitas periódicas alrededor de $\mathrm{P}_{\mathrm{e}}$ vía bifurcaciones de Hopf. La transversalidad se verifica con la derivada respecto al parámetro $\mathrm{A}$, es decir :

$$
\frac{\partial(\text { trMe })}{\partial A}=-(1-C)<0, \text { cuando } A^{*}=\frac{1-C}{1+C}
$$

es el valor de bifurcación. Se tiene que, sobre la superficie 1 - C - A - A C $=0$, en el espacio de parámetros $\mathrm{Q}_{\mathrm{e}}$, el punto de equilibrio $\mathrm{P}_{\mathrm{e}}$, situado en el primer cuadrante es un centro-foco débil.

\section{REFERENCIAS}

\section{Amundsen, E. S., T. Bjørndal y J. M. Conrad. 1995.} Open access harvesting of the northeast atlantic minke whale. Envir. Res. Econ., Dordrecht, 6: 16785 .

Andronov A. A., E. A. Leontovich, I. I. Gordon, y A. G. Maier. 1973. Qualitative theory of secondorder dynamic systems. John Wiley and Sons, New York, $524 \mathrm{pp}$.

Ardito, A. , P. Ricciardi y A. Schiaffino. 1993. The effects of seasonal fluctuations of an open access fishery problem. J. Math. Biol., Berlin, 31: 879-
890.

Arrowsmith, D. K. y C. M. Place. 1992. Dynamical systems: differential equations, maps and chaotic behaviour. Chapman and Hall, London, 330 pp.

Bjørndal, T. y J. M. Conrad. 1987. The dynamic of an open access fishery. Can. J. Econ. XX (1): 74-85.

Cheng K-S., S-B. Hsu y S-S. Lin. 1981. Some results on global stability of a predator-prey systems. J. Math. Biol., Berlin, 12: 115-126.

Clark, C. W. 1974. Mathematical bioeconomics. In: P. van den Driesche (ed.) Mathematical Problems in Biology (Victoria Conference). Lecture Notes in Biomathematics 2, Springer-Verlag, Berlin, pp 29-45.

Clark, C. W. 1979. Mathematical models in the economics of renewable resources. SIAM Rev. 21 (1): $81-89$

Clark, C. W. 1985. Bioeconomic modelling and fisheries managements. John Wiley and Sons, New York, $291 \mathrm{pp}$.

Clark, C. W. 1990. Mathematical bioeconomics. The optimal management of renewable resources (2nd ed). John Wiley and Sons, New York, 386 pp.

Conrad, J. y C. W. Clark. 1987. Natural resources economics: notes and problems. Cambridge University Press, Cambridge, 231 pp.

Goh, B-S. 1980. Managment and analysis of biological populations. Elsevier Scientific Publishing Company, Amsterdam, 279 pp.

González-Olivares, E. 1991. El modelo de Smith para una pesquería de acceso abierto. Una revisión crítica. An. Inst. Mat., U. Cat. Valp., Valparaíso: 83-92.

González-Olivares E. y J. Mena-Lorca. 1994. Análisis cualitativo de un modelo de pesquerías de acceso abierto. Invest. Mar., Valparaíso, 22: 3-11.

González-Olivares E y S. Osorio-Santibáñez. 1997. Una clase de modelos bioeconómicos continuos con captura tipo Cobb-Douglas. Mem. Oct. Congr. Inter. Biomat., Panamá: 147-155.

Gordon, H. S. 1954. Economic theory of a commonproperty resources: the fishery. J. Pol. Econ. 62: 129 - 142. Reprinted in Bull. Math. Biol. Vol. 53, (1/2): 231-252. 1991.

Hannesson, R. 1993. Bioeconomic analysis of 
fisheries. Fishing New Books, Oxford, 138 pp.

Hilborn, R. y C. J. Walters, 1992. Quantitative fisheries stock assesment: choice, dynamic and uncertainty. Chapman and Hall, London, 542 pp.

Koçak, H. 1986. Differential and difference equations through computer experiments. Springer-Verlag, Berlin, 225 pp.

May, R. M. 1974. Stability and complexity in model ecosystems. Princenton University Press, Princenton, $261 \mathrm{pp}$.

Murray, J. D. 1989. Mathematical biology. SpringerVerlag, Berlin, 767 pp.

Opsomer, J-D. y J. M. Conrad. 1994. An open-access analysis of the northern anchovy fishery. J. Envir. Econ. Man. 27: 2-37.

Pella, J. J. y P. K. Tomlinson. 1969. A generalized stock production model. Bull. Inter-Am. Trop. Tuna Comm. 13: 421-496.

Rothschild, B. J. 1977. Fishing effort. In: J. A. Gulland (ed.) Fish population dynamics. A Wiley-

Recibido el 7 de enero de 1997.

Aceptado el 2 de julio de 1998.
Interscience Publication, New York, pp. 96-115.

Sánchez-Garduño, F. 1990. La respuesta y la coexistencia en algunos modelos ecológicos. Rev. Sem. Ens. Tit., UNAM., Ciudad de México Vol. VI (49), $22 \mathrm{pp}$.

Schaefer, M. B. 1954. Some aspects of the dynamics of population important to the management of the commercial marine fisheries. Bull. Inter-Am. Trop. Tuna Comm. 1 (2): 27-56. Reprinted in Bull. Math. Biol. 53, (1/2): 253-279. 1991.

Smith V. L. 1969. On models of commercial fishing. J. Polit. Econ. 77: 181-198.

Sotomayor, J. 1979. Liçoes de equaçoes diferenciais ordinarias. IMPA Projecto Euclides, Rio de Janeiro, 327 pp.

Spencer, P. L. 1997. Optimal harvesting of fish populations with nonlinear rates of predation and autocorrelated environmental variability. Can. J. Fish. Aquat. Sci. 54: 59-74.

Walters, C. J. 1986. Adaptive management of renewable resources. Macmillan Publishing Company, New York, 366 pp.

Yodzis, P. 1989. Introduction to theoretical ecology. Harper and Row, New York, 384 pp. 